\title{
Influence of Mini-Scleral Contact Lens Wear on Cornea in Ectatic Corneal Disorders
}

\section{IJCRR \\ Section: Healthcare ISI Impact Factor (2020-21): 1.899 IC Value (2020): 91.47 SJIF (2020) $=7.893$ \\ (c) (i) (8) Copyright@IJCRR}

\section{Simi Afroz ${ }^{1}$, Khowal Ritesh ${ }^{2}$, Dubey Gaurav ${ }^{3}$, Jamshedi Ali4*, Yadav Juhi5, Das Prasenjit', Manik K. R ${ }^{7}$}

\begin{abstract}
'Assistant Professor, Department of Optometry, School of Allied Health Sciences, Sharda University, New Delhi, India; ${ }^{2}$ Consultant Optometrist, Dr R.P Center AllMS, New Delhi, India; ${ }^{3}$ Optometry Resident, Department of Optometry, FPS, Uttar Pradesh University of Medical Sciences, Saifai, Etawah, India; ${ }^{4}$ Assistant Professor, Department of Optometry, Allied Health Science IIMT University, Meerut, Uttar Pradesh, India; ${ }^{5}$ Assistant Professor, Department of Allied Health Science, TMU University, Moradabad, Uttar Pradesh, India; ${ }^{6}$ Assistant Professor, Department of Allied Health Science, ITM University, Gwalior, India; ${ }^{7}$ Assistant Professor, Department of Paramedical Science, Subharti University, Meerut, India.
\end{abstract}

\section{ABSTRACT}

Introduction: Scleral lenses are gaining popularity in places where contact lenses are fitted. MSCL's sub-group of scleral contact lenses with a total lens diameter between 14 and $18 \mathrm{~mm}$, which rest entirely upon the sclera.

Aim: The study aims to evaluate the influence of mini-scleral contact lens (MSCL) wear on corneal shape, thickness, and higherorder aberration after the lens settled on the eye for 4 to 6 hours in non-inflammatory ecstatic corneal disorders.

Purpose: The study aims to check the change in corneal curvature and thickness after settling the lens on the eye for 4 to 6 hours. Also, check if there is any change in corneal aberration just after insertion and after settling the lens for 4 to 6 hours.

Methods: This study was a prospective Cross-sectional experimental study to prove what was hypothesized in advance. Scheimpflug imaging was done for 15 subjects (mean age $26.4( \pm 6.57)$ ), first before insertion of mini-scleral contact lens. Second, just after wearing the mini-scleral contact lens. Third, after 4 to 6 hours of wearing of mini-scleral contact lens. Fourth, just after removal of MSCL in non-inflammatory ecstatic corneal disorder patient. Natural diurnal variations were considered by measuring separate control groups.

Results: Small but significant increase in corneal thickness was observed just after removal of the lens after 4 to 6 hours of wearing (from $447.2( \pm 46.8)$ to $457.8( \pm 50.35) \mu$ ) $\mathrm{P}=0.023$. After 4 to 6 hours of wearing, significant flattening of steep and steepest corneal curvature was observed having mean $(0.0524( \pm 0.085) \mathrm{mm}, 0.933( \pm 1.6) \mathrm{D}$ respectively $(P=0.032)$.

Conclusion: Increase in corneal thickness, and significant flattening of steep corneal curvature was observed immediately after removing the lens after 4 to 6 hours of wearing. Modern MSCL decreases both higher and lower order aberration immediately after wearing the lens. Modern mini scleral lenses influence the corneal shape and induce small but significant corneal oedema after 4 to 6 hours of wearing.

Key Words: Mini scleral contact lens (MSCL), Corneal Aberrations, Corneal thickness, Ecstatic cornea, Keratoconus, RGP Contact Lens

\section{INTRODUCTION}

Scleral lenses are gaining popularity in places where contact lenses are fitted. MSCL's a sub-group of scleral contact lenses with a total lens diameter between 14 and $18 \mathrm{~mm}$, which rest entirely upon the sclera, is sealed to the anterior eye with minimal movement upon blinking or ocular versions (Sindt, 2008). ${ }^{1}$ They are primarily used to correct irregular corneal optics commonly encountered in Keratoconus, Keratoglobus, Post LASIK Ectasia, as the post-lens tear layer (the fluid reservoir between the posterior lens and anterior cor- nea) effectively neutralizes most of the corneal astigmatism. More recently, scleral lenses have also been utilized as a therapeutic intervention in cases of ocular surface disease (e.g., Exposure Keratopathy, Sjogren's syndrome, StevenJohnson's Syndrome) as they provide hydration to the cornea during lens wear without evaporation. Previously, scleral lenses were larger in diameter, and the fitting was empirical and primarily relied upon practitioner interpretation of haptic (landing zone) vascular compression of the bulbar conjunctiva. Newer technology and advancements in anterior

Corresponding Author:

Jamshedi Ali, Assistant Professor, Department of Optometry, Allied Health Science, IIMT University, Meerut, Uttar Pradesh, India. Email: alijamshed429@gmail.com

ISSN: 2231-2196 (Print)

Received: 22.09 .2021
ISSN: 0975-5241 (Online)

Revised: 16.10 .2021
Accepted: 09.11.2021 
eye imaging with corneal topography and optical coherence tomography have resulted in a more reliable and accurate fitting process. Improved lens design led to the increase in the prescription of scleral lenses. This has led to a subsequent increase in the scleral contact lens prescription. Scleral lenses are significantly thicker (up to $1300 \mu$ central thickness for full scleral lenses) than corneal rigid gas permeable (RGP) lenses to avoid the eye and handling flexure. Consequently, to counteract this increased thickness, modern scleral lenses are manufactured from highly permeable materials to maximize oxygen transmission to the cornea (Vincent, 2014). ${ }^{2}$ This is particularly important since scleral lenses do not move upon blinking to allow freshly oxygenated tears to replenish the post-lens tear layer.Post-lens tear thickness varies from lens to lens, cornea to cornea and practitioner to practitioner. It may act as an additional barrier to the corneal oxygen reaching the atmosphere. The increased lens thickness, poor tear exchange and the presence of a thick postlens tear layer are all possible hypoxia variables, however clinical reports of substantial corneal oedema associated with current scleral lens usage are rare. There have been several investigations into the corneal response to scleral lens wear in an attempt to quantify it. In an early study using full scleral lenses, Bleshoy and Pullum reported corneal oedema in a single subject following $5 \mathrm{hrs}$ of sealed scleral contact lens wear using an RGP material with a Dk of 24. Central lens thickness was varied from 180 to $500 \mu$, and corneal swelling increased slightly with increasing lens thickness from $3.6 \%$ to $4.8 \%$ centrally and $4.6-5.3 \%$ in the periphery (although these central and peripheral zones were not defined). More recently, Pullum and Stapleton assessed central corneal swelling for four subjects following 3 hours of sealed scleral lens wear while varying lens thickness and oxygen permeability. Sclera lenses with a thickness of 1200 and a Dk of 32 showed up to $8 \%$ corneal edema, although this decreased as the lens thickness was reduced and the Dk increased. Other studies have looked at how well scleral contact lenses fit (such as apical clearance) and how they affect vision and ocular health (such as enhanced acuity, lens tolerance, and problems).This study assessed the physiological changes in corneal characteristics (biometrics and optical properties including anterior higher order aberrations) associated with modern MSCL wear. Scheimpflug imaging was used to investigate the influence of MSCL wear on the irregular cornea, young subjects with the ecstatic non- inflammatory irregular cornea (i.e., keratoconus or corneal abnormalities including ocular surface disease) over a substantially larger corneal region than previously examined. There are some drawbacks to correcting higher-order aberrations in normal eyes, including the fact that the eye is a dynamic optical and biological system. Consequently, the optical aberrations measured and corrected one day may not yield the same level of visual improvement on another day (Jinabhai, 2009). ${ }^{3}$ Jinabhai study also reports that aberration measurements can come from accommodation fluctuations, tear film fluctuations, small eye movements and pupil size changes (Jinabhai, 2009). ${ }^{3}$ Simply said, correcting aberrations in normal eyes is like correcting a little fluctuating value. Despite the disadvantages for normal eyes, higher-order aberration contact lenses could improve vision in ecstatic eyes due to the huge magnitudes of optical aberration present in these patients. The practicality of ecstatic eye contact lenses with aberration control is being studied. Correction of these optical imperfections would greatly improve joyful eyes' visual performance. The first step is to precisely measure sick optical defects. To explain the optical distortions, present in the ecstatic cornea. It will also assess the progress made in correcting them with micro scleral contacts. Currently, these tailored lenses only partially correct higher-order aberrations in keratoconus eyes. The use of aberrationcontrolling contact lenses in non-inflammatory ecstatic eyes is investigated in this research. Currently, aberration-controlling contact lenses seem realistic. If designers can create bespoke lenses with precision and movement stability. The study aims to estimate the change in corneal curvature and thickness after settling the MSCL on eye for 4 to 6 hours and to evaluate the change in aberration after settling the lens for 4 to 6 hours. The Stephen study concludes that Modern miniscleral contact lenses that vault the cornea may slightly influence corneal shape and power but do not induce clinically significant corneal oedema during short-term wear. (Stephen J. Vincent, 2014). ${ }^{3,4}$ Pullum says in his study that the calculations of oxygen transmissibility, with varying tear layer and lens thicknesses, ranged from 10 to 36.7 at the scleral lens centres and from 17.4 to 62.6 at the peripheries. Their calculations of maximum central lens thicknesses show a practical range of $250-495 \mu \mathrm{m}$, in conjunction with a post-lens tear layer thickness of 100-250 $\mu \mathrm{m}$. He also used the HoldenMertz Dk/t criteria of 24 Fatt units for the central cornea. The Harvitt-Bonanno criteria of 35 Fatt units for the limbal area were used as reference points (Pullum, 1997). ${ }^{5}$ Article by customized lenses is found to correct only partially the higher-order aberrations found in keratoconic eyes. Suppose contact lens designers can manufacture customized lenses with accuracy and good movement stability. In that case, aberration-controlling contact lenses could prove to be a very useful tool for those involved in managing keratoconus patients (keratoconus, 2015). ${ }^{6}$ Jinabhai said the use of MSCL correcting higher-order aberrations may still be extremely beneficial in improving visual function in keratoconic eyes due to the enormous magnitudes of optical aberration observed in these patients. (Jinabhai, 2009). ${ }^{3}$ Alio J. L., in his study, shows that the eyes with the lenses have a statistically significantly thicker cornea compared to the non-lens wearing eye after wearing either lens for 8 hours, lying within clinically and physiologically acceptable limits. Their clinical findings contradict existing theoretical estimates, which predict an increase in corneal edema as the tear layer thickness 
increases.It has to be evaluated if the effect on corneal oedema changes with longer wearing periods, larger samples, or other influences (Alió J. L., Corneal higher order aberrations: a method to grade keratoconus, 2006). ${ }^{6,7}$ Christiane Arlt's Despite current theoretical estimates, clinical evidence show that increased tear layer thickness does not increase corneal edema.It has to be evaluated if the effect on corneal oedema changes with longer wearing periods, larger samples, or other influences (Christiane Arlt, 2015). ${ }^{8}$ As far as my knowledge ours is the only study done on young, healthy corneas but not on irregular or ecstatic corneas. Also, there is no study on aberration change after settling the lens for 4 to 6 hours. The study's main objective is to estimate the change in corneal curvature and thickness after settling the lens on eye for 4 to 6 hours and to check the change in aberration before and after insertion of the lens.

\section{METHODOLOGY}

This study was a prospective Cross-sectional experimental study to prove what was hypothesized in advance. The author collected the data according to the hypothesis. The prevalence of the ecstatic corneal disorder is very low. (2300/100,000 for keratoconus) (Jonas, 2009). ${ }^{9}$ So sample size collection was based on convenient sampling. This study's inclusion criterion was Irregular cornea (ecstatic non-inflammatory corneal disorders); all subjects were between the ages of $18 \mathrm{yrs}$. to $50 \mathrm{yrs}$. with both males and females, and Post collagen cross-linking were also included. All kinds of Systemic Diseases, Presence of ocular disease except for non-inflammatory ecstatic corneal disorders), Age Group above 50 years, Age Group below 18 years, those who were absent on the day of the study, those who did not consent to participate in the study were excluded from this study All the patients presented in the visual aids centre Lajpat Nagar New Delhi were included in our study. They all took part voluntarily and without a financial refund. Ten subjects were included in the study. The average age within the sample was 34.5 years, with a standard deviation of ${ }^{11} 06$ years at the time of the measurements. The consent form was signed, which shows their voluntary participation in my study. All the subjects had to pass the pre-fitting evaluation; the ideal diameter and base curve were chosen using the provided fitting set. If evaluation with fluorescein at the slit lamp did not look as expected, modifications were made in cooperation with the manufacturer. After finalizing the lens parameters, the lens was ordered with final refractive power incorporated in the lens. The vault used in the study is $300 \pm 25$ micron. To avoid diurnal variation, the subject had to wear the lens in the morning at about 10:00 am and remove after 4 to 6 hours, about 3:00 pm. Only if all requirements were fulfilled, the lens could be used in the study. Data were coded and recorded in the MS Excel spreadsheet program. SPSS v16 was used for data analysis. Shapiro-Wilk normality test, pairedsample t-test, and Wilcoxon sign ranked test were used according to suitability and analysed data. The level of significance was taken as $p<0.05$. Various graphs were used for showing the pre vs post changes of each parameter. The prior approval was taken by the hospital authority to conduct this.

\section{Table 1}

\begin{tabular}{|l|}
\begin{tabular}{|l|} 
Four scans were taken in the following Sequence of the \\
Measurement:
\end{tabular} \\
\hline First, before fitting the mini-scleral contact lens. \\
\hline Second, just after fitting the mini-scleral contact lens. \\
\hline $\begin{array}{l}\text { Third, after } 4 \text { to } 6 \text { hours of wearing of mini-scleral contact } \\
\text { lens. }\end{array}$ \\
\hline Fourth, just after removal of the mini- scleral contact lens. \\
\hline $\begin{array}{l}\text { Above mentioned parameters were measured. Visual } \\
\text { acuity was measured five times using a log MAR chart in } \\
\text { the following way }\end{array}$ \\
\hline $\begin{array}{l}\text { First unaided visual acuity was measured before fitting the } \\
\text { contact lens and also without any refractive error. }\end{array}$ \\
\hline Second, just after fitting the final lens. \\
\hline Third after 4to 6 hours of wearing the lens. \\
\hline $\begin{array}{l}\text { Fourth unaided visual acuity was measured just after removal } \\
\text { of the lens. }\end{array}$ \\
\hline
\end{tabular}

\section{RESULT}

Fifteen (15) subjects (12 male and 3 Female) of mean age $26.4( \pm 6.57)$. The mean apex pachymetry (micron) increased from 447.2( \pm 46.476$)$ before insertion of MSCL to $457.8( \pm 50.35)$ after removal of the lens after approx. 5hours. This change was statistically significant (Paired SampleTest), 'p' $=0.023$ (Figure- 1).

The mean of Thinnest corneal thickness before insertion of MSCL increase from $431( \pm 38.46)$ to $443( \pm 44.34)$ after approx. 5 hours. This change was significant paired-sample ttest), 'p'=0.013 (Figure-2).

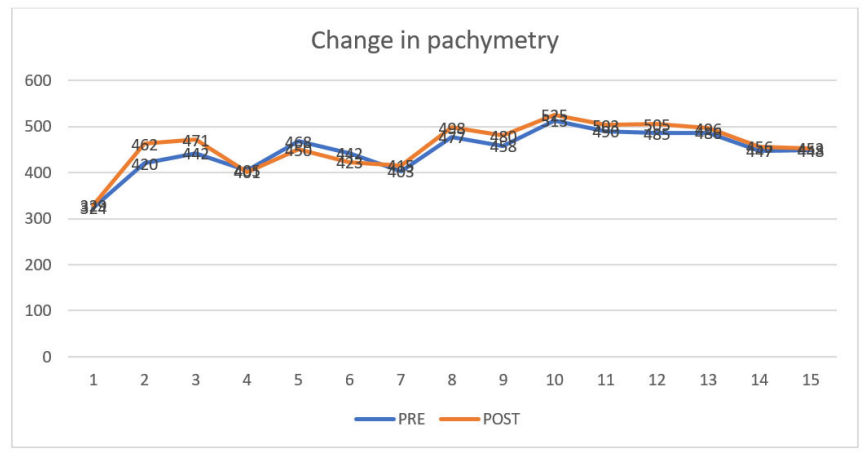

Figure 1: Individual change in apex pachymetry. 


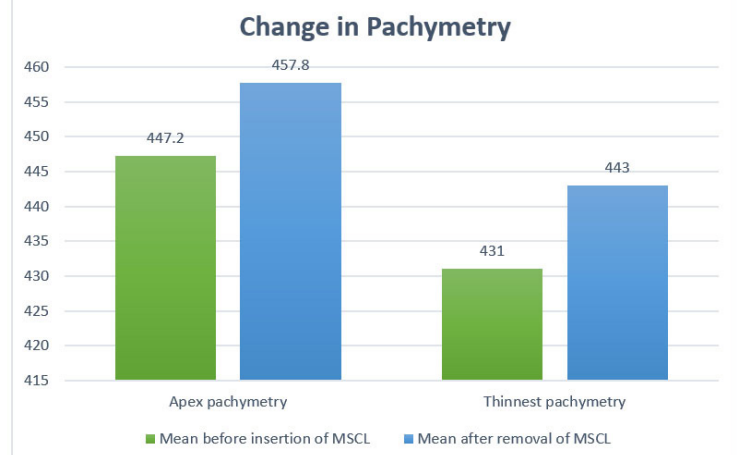

Figure 2: Mean change in pachymetry of both apex and thinnest.

There is no significant change in the flat corneal curvature before insertion of mini scleral and just after removal of MSCL. But there is a significant change in steep corneal curvature before insertion, and just after removal of MSCL with ' $p$ ' $=0.032$, mean value of steep corneal curvature before insertion of MSCL and just after removal of mini sclera contact lens is $6.55( \pm 0.78)$ and $6.61( \pm 0.082)$ respectively (Figure-3).

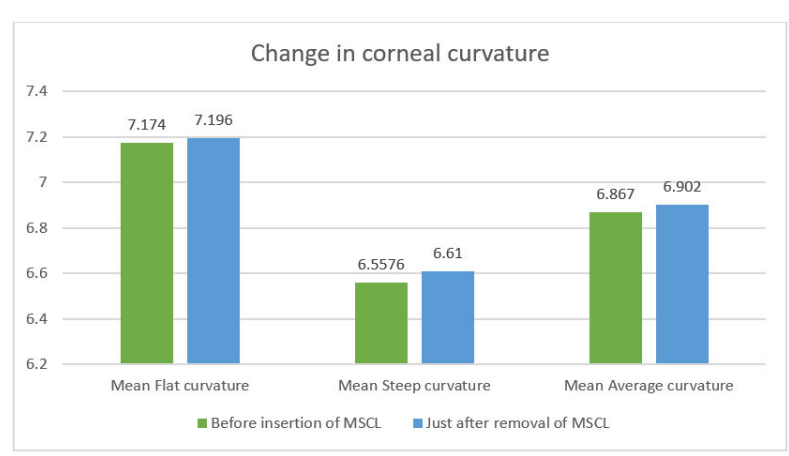

Figure 3: Mean change in corneal curvature.

There was also a significant change in the steepest corneal curvature before insertion and just after removal of MSCL with ' $\mathrm{p}$ ' $=0.049$ (Figure-4). There is a flatting of approximately $0.933( \pm 1.6)$ in the steepest corneal curvature. The mean value of the steepest corneal curvature before insertion of MSCL and just after removal of MSCL are 58.57( \pm 7.76$)$ and $57.64( \pm 7.85)$, respectively. There were no significant changes in Pmax, variance index, and irregularity index with ' $\mathrm{p}$ '= $0.158,0.619$ and 0.426 , respectively (Table-1).

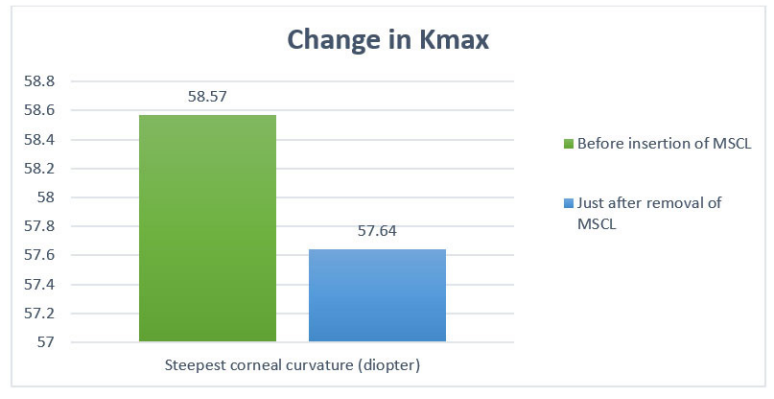

Figure 4: Change in steepest corneal curvature.
Table 1: Mean value and p-value of pre and post corneal curvature and thickness

\begin{tabular}{|c|c|c|c|c|}
\hline \multirow{2}{*}{$\begin{array}{l}\text { Sr. } \\
\text { No. }\end{array}$} & \multirow{2}{*}{ Parameters } & \multicolumn{2}{|c|}{ Mean value } & \multirow[t]{2}{*}{ p-value } \\
\hline & & $\begin{array}{c}\text { Before insertion } \\
\text { of MSCL }\end{array}$ & $\begin{array}{l}\text { After removal } \\
\text { of MSCL }\end{array}$ & \\
\hline 1. & $\begin{array}{l}\text { Apex pachym- } \\
\text { etry (micron) }\end{array}$ & $447.2( \pm 46.8)$ & $457.8( \pm 50.35)$ & 0.023 \\
\hline 2. & $\begin{array}{l}\text { Thinnest } \\
\text { pachymetry } \\
\text { (micron) }\end{array}$ & $431.8( \pm 38.46)$ & $443( \pm 44 \cdot 34)$ & 0.013 \\
\hline 3. & $\begin{array}{l}\text { Flat corneal } \\
\text { curvature }(\mathrm{mm})\end{array}$ & $7.17( \pm 0.79)$ & $7.19( \pm 0.82)$ & 0.593 \\
\hline 4. & $\begin{array}{l}\text { Steep corneal } \\
\text { curvature }(\mathrm{mm})\end{array}$ & $6.55( \pm 0.78)$ & $6.61( \pm 0.78)$ & 0.032 \\
\hline 5. & $\begin{array}{l}\text { Average corneal } \\
\text { curvature }(\mathrm{mm})\end{array}$ & $6.86( \pm 0.78)$ & $6.90( \pm 0.79)$ & 0.228 \\
\hline 6. & Kmax(dioptre) & $58.57( \pm 7.76)$ & $57.64( \pm 7.85)$ & 0.049 \\
\hline 7. & $\begin{array}{l}\text { Power } \\
\max (\text { dioptre) }\end{array}$ & $55 \cdot 71( \pm 7.18)$ & $54.97( \pm 7.18)$ & 0.158 \\
\hline 8. & $\begin{array}{l}\text { Index of vari- } \\
\text { ance }\end{array}$ & $92.93( \pm 34.058)$ & $92.13( \pm 33.89)$ & 0.619 \\
\hline 9. & $\begin{array}{l}\text { Irregularity } \\
\text { index }\end{array}$ & $0.0622( \pm 0.0658)$ & $0.0418( \pm 0.020)$ & 0.426 \\
\hline
\end{tabular}

\section{Relation between before insertion and just after insertion of MSCL:}

RMS of total aberrations before insertion of MSCL decreases from $10.397( \pm 3.70)$ to $4.67( \pm 3.05)$ (mean value) just after insertion of MSCL with ' $p$ ' $=0.001$, which is highly significant (Figure-5).

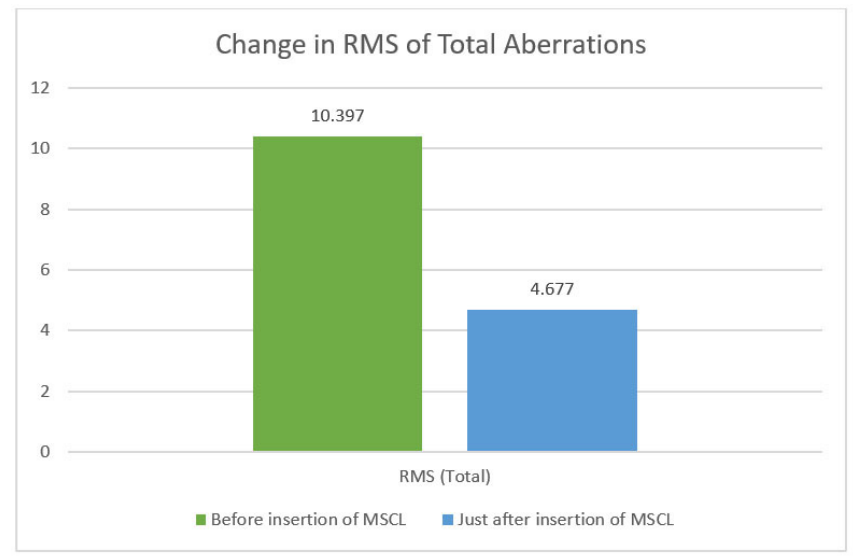

Figure 5: Change in RMS of total Aberration.

RMS of LOA aberration before insertion of MSCL also decreases from $10.08( \pm 3.57)$ to $4.87( \pm 2.70)$ (mean value) just after insertion of MSCL, which is statistically significant with ' $\mathrm{p}$ ' $=0.000$. RMA of HOA decreases before insertion of MSCL from $2.75( \pm 1.16)$ to $1.3873( \pm 0.92)$ (mean value) just after insertion of MSCL, which is statistically significant with ' $p$ ' $=0.001$. Spherical aberration before insertion of MSCL decreases from $0.756( \pm 0.76)$ to $0.392( \pm 0.34)$ (mean 
value) just after insertion of MSCL with 'p' $=0.017$, which is statistically significant. Secondary astigmatism before insertion of MSCL decreases from 2.88( \pm 2.21$)$ to 2.013( \pm 2.76$)$ just after insertion of MSCL with ' $\mathrm{p}$ ' $=0.020$, which is statistically significant. Defocus before insertion of MSCL decreases from $3.362( \pm 2.95)$ to $1.33( \pm 1.30)$ just after insertion of MSCL with significant ' $p$ ' $=0.003$. Trefoil aberration before insertion of MSCL decreases from $0.408( \pm 0.29)$ to $0.332( \pm 0.35)$ just after insertion of MSCL with ' $\mathrm{p}$ ' $=0.001$, which is highly statistically significant. Coma aberration before insertion of MSCL decreases from $0.85( \pm 1)$ to $0.627( \pm 0.74)$ just after insertion of MSCL with ' $p$ ' $=0.036$, which is statistically significant. Quadra foil, Penta foil, and hexafoil did not show statistically significant results with 'p' $=0.394,0.443$ and 0.609 (Figure-6).

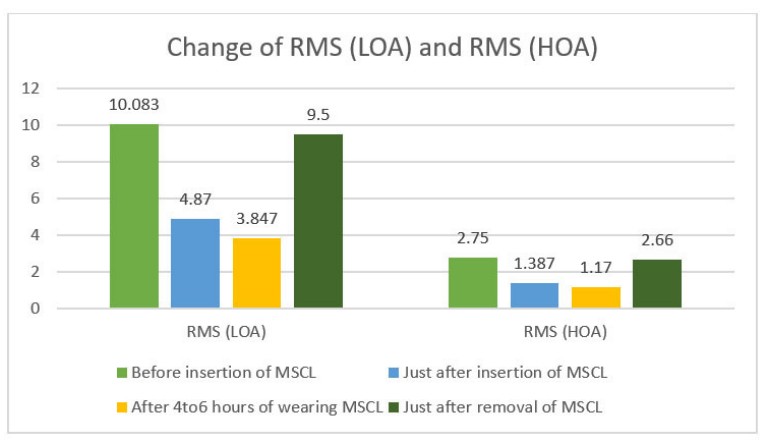

Figure 6: Change in RMS of LOA and HOA.

\section{Relation between just after insertion and be- fore removal after $\mathbf{4}$ to $\mathbf{6}$ hours:}

RMS of HOA further decreases from $1.387( \pm 0.92)$ to $1.17( \pm 0.87)$ (mean value) with ' $p '=0.047$ which is statistically significant. RMS of LOA further decreases from $4.87( \pm 2.7)$ to $3.847( \pm 2.29)$ with a ' $\mathrm{p}$ ' $=0.036$ which is statistically significant (Table-2). Secondary astigmatism further decreases from $2.013( \pm 2.76)$ to $1.107( \pm 0.95)$ with a 'p' $=0.044$ which is statistically significant. Spherical aberration, Defocus, Trefoil, Coma, Quadra foil, Penta foil and Hexa foil did not show any statistically significant result having 'p' $=0.82,0.394,0.334,0.191,0.307,0.307$ and 0.293 , respectively (Figure-7).

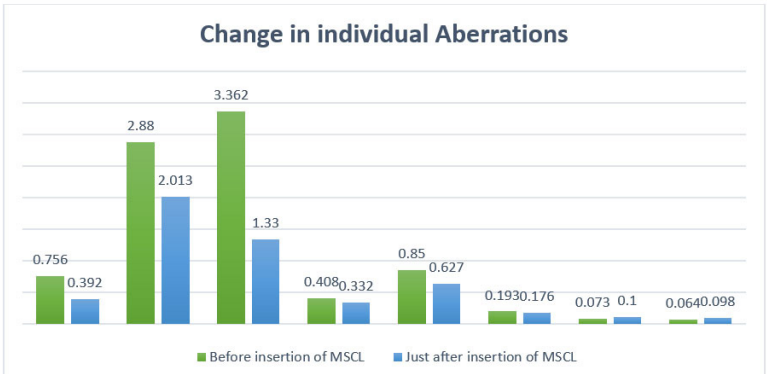

Figure 7: Change in the mean of individual Aberrations.
Table 2: p-value of aberrations in different pair

$\begin{array}{lccc}\begin{array}{l}\text { Types of } \\ \text { aberrations }\end{array} & \begin{array}{c}\text { Before insertion } \\ \text { and just after } \\ \text { insertion of } \\ \text { MSCL }\end{array} & \begin{array}{c}\text { Just after } \\ \text { insertion } \\ \text { and after } 4 \\ \text { to } 6 \text { hours } \\ \text { of MSCL } \\ \text { wear }\end{array} & \begin{array}{c}\text { Before in- } \\ \text { sertion and } \\ \text { just after } \\ \text { removal of } \\ \text { MSCL }\end{array} \\ \text { RMS (total) } & 0.001 & 0.801 & 0.061 \\ \text { RMS(LOA) } & 0.000 & 0.047 & 0.140 \\ \text { RMS(HOA) } & 0.001 & 0.036 & 0.061 \\ \text { Spherical } & 0.017 & 0.820 & 0.776 \\ \text { aberration } & 0.020 & 0.044 & 0.182 \\ \text { Secondary } & 0.003 & 0.394 & 0.281 \\ \text { astigmatism } & 0.001 & 0.334 & 0.925 \\ \text { Defocus } & 0.036 & 0.191 & 0.570 \\ \text { Trefoil } & 0.394 & 0.307 & 0.955 \\ \text { Coma } & 0.443 & 0.307 & 0.620 \\ \text { Quadra foil } & 0.609 & 0.609 & 0.496 \\ \text { Penta foil } & & & \\ \text { Hexa foil } & & & \end{array}$

\section{Before insertion and just after removal of MSCL:}

There is no statistically significant change in any aberration before insertion and just after removal of MSCL after 4 to 6 hours of wearing.

\section{DISCUSSION}

Our best knowledge is the only Indian study that reveals statistically significant corneal oedema following 4 to 6 hours wearing of MSCL. There is an increase in central corneal thickness following 4 to 6 hours of wearing MSCL with the mean percentage change of 0.0236 , whereas Stephen Vincent et al. study says the opposite. The increase in corneal thickness is either due to hypoxia or the imbibition of saline used in MSCL or it may be a combination of both. To minimize saline imbibition, we use near equivalent composition, osmolarity, $\mathrm{pH}$, and refractive index as that of tear layer. (Michaud, 2012) ${ }^{10}$ in his study concluded that scleral contact lens with Dk of 100 and lens thickness of $300 \mu \mathrm{m}$ and corneal vault of $400 \mu \mathrm{m}$ would yield a predicted $\mathrm{Dk} / \mathrm{t}$ of 12.5, which do not satisfy Holden and Merts criteria for successful daily wear, so the clinically or statistically significant corneal swelling was observed (Michaud, 2012). ${ }^{10}$ There is a flatting of steep corneal curvature following 4 to 6 hours of MSCL wear. Still, no significant change was noted in flat corneal curvature, whereas Vincent et al. study says overall flatting of corneal curvature. There is also a flatting of steepest corneal curvature $(0.035( \pm 0.1))$ following 4 to 6 hours of MSCL wear. Analysis of change in individual Zernike coefficient up to 6 th order aberration was done. But 
only up to 4 th order aberration shows a significant change in before and immediately following MSCL wear. When we take individual aberration into account, there is no further change after wearing 4 to 6 hours of MSCL except RMS of HOA, RMS of LOA, and secondary astigmatism shows a statistically significant decrease. RMS to LOA shows a highly statistically significant decrease in aberration. There is numerous higher-order aberration of which only spherical aberration, coma and trefoil are of clinical interest. Spherical aberration is commonly increased in myopic LASIK, surface ablation and keratoconus (non-inflammatory ecstatic corneal disorder (Michaud, 2012). ${ }^{10}$ Spherical aberration results in halos around the point image. It is statistically decreased in the ecstatic cornea after wearing MSCL. Coma is common in patients with decentred corneal graft, keratoconus, and decentred laser ablations (Michaud, 2012). ${ }^{10}$ Our study shows a statistically significant decrease in coma aberration after wearing MSCL. Trefoil produces less degradation in image quality compare with coma. It causes glare, ghost images and loss of contrast. They make up to about $15 \%$ of the total number of aberrations in an eye. (Vessel, 2020). ${ }^{11}$ LOA account for approx. $85 \%$ of the overall wave aberration in the eye. It includes positive defocus, negative defocus, and regular astigmatism. LOA mainly accounts for visual acuity, i.e., Measure of form sense. Our study shows a highly significant decrease in RMS of LOA ( $p$-value $=0.000$ ) and the same we get by measuring visual acuity before and after insertion of MSCL ( $\mathrm{p}$ ' $=0.001$ ). There is a statistically significant decrease in defocus before insertion and just insertion of MSCL ('p'=0.003). RMS of LOA and RMS of HOA further decrease 3 hours after lens wear. Individual aberration remains content and no further change, showing that MSCL did not affect the corneal aberration after wearing short-term MSCL (Vessel, 2020). ${ }^{11}$ There is no evidence of change in corneal aberration before insertion of MSCL and just after removal of MSCL after 4 to 6 hours of wear, which indicates that the lens does not cause any significant change in LOA. There is no evidence of change in corneal aberration just after insertion of MSCL and before removal of MSCL after 4 to 6 hours of wearing, which indicates mini scleral. This particular type of lens provides stable constant vision. Different lens materials and designs should be taken into consideration (Vessel, 2020). ${ }^{11}$

\section{CONCLUSION}

Corneal thickness has changed and increased after settling the MSCL on the eye for 4 to 6 hours. There is flattening of steep corneal curvature and the steepest corneal curvature (Kmax) after settling the MSCL on the eye for 4 to 6 hours. There is no evidence of change in flat corneal curvature. There is a decrease in total aberration after wearing the lens, and there is no evidence of a change in aberration after settling the lens for 4 to 6 hours of wearing. There is an increase in visual acuity just after wearing the lens. Modern MSCL decreases both higher and lower order aberration immediately after wearing the lens. Modern mini scleral lenses influence the corneal shape and induce small but significant corneal oedema after 4 to 6 hours of wearing.

\section{Limitations}

This study was a prospective Cross-sectional experimental study; further research on a more diverse group in terms of keratoconus, PMCD, post LASIK is required to arrive to reach a strong conclusion and recommendation.

\section{Smaller sample size.}

Lack of human resources and lack of awareness among practitioner and patient.

\section{Recommendation}

We recommend a similar study with a larger sample size, and a more diverse group should be considered to ascertain that similar findings will be revealed.

\section{ACKNOWLEDGEMENT}

The authors would like to thank the higher authorities of the visual aids centre Lajpat Nagar New Delhi for providing all possible support for the smooth conduction of this research. The authors acknowledge the immense help received from the scholars whose articles are cited and included in references to this manuscript. The authors are also grateful to authors/editors/publishers of all those articles, journals, and books from where the literature for this article has been reviewed and discussed."

\section{Conflict of Interest}

This research did not receive any outside funding or support

\section{Source of funding-None}

\section{Ethical Statement-}

The prior approval was taken by the hospital authority to conduct this.

\section{Authors' Contribution:}

\section{Abbreviations:}

1. MSCL- mini scleral contact lens

2. MAR- minimum angle of resolution

3. SPSS- Statistical Package for the social sciences

4. RMS- root mean square

5. LOA- lower order aberration

6. HOA-higher order aberration

7. PMCD- pellucid marginal corneal degeneration

8. LASIK- laser assisted in situ keratomileusis 


\section{REFERENCES}

1. Sindt C. Basic scleral lens fitting and design. Contact Lens Spectrum. 2008; 23(10), 10.

2. Vincent S. J.-C.Corneal changes following short-term miniscleral contact lens wear. contact lens and anterior eye.2014; 37(6), 461-468.

3. Jinabhai A. R. Higher order aberrations in keratoconus: A review. optom. pract. 2009

4. Stephen J. Vincent, D. A.-C. Corneal changes following shortterm miniscleral contact lens wear. Contact Lens \& Anterior Eye. 2014 37, 461-468.

5. Pullum, K. W. Scleral lens induced corneal swelling: what is the effect of varying Dk and lens thickness? The CLAO journal: official publication of the Contact Lens Association of Ophthalmologists, Inc.1997; 23(4), 259-263.

6. Keratoconus, O. E. The Epidemiology and aetiology of keratoconus. International journals of keratoconus and ectatic corneal disease.2015; 7-15.
7. Alió, J. L. Corneal higher order aberrations: a method to grade keratoconus. Journal of Refractive Surgery.2006; 22(6), 539545.

8. Christiane Arlt, P. P.Clinical Effect of Tear Layer Thickness on corneal edema during scleral lens wear. alien university.2015; $1-79$.

9. Jonas, J. B. Prevalence and associations of keratoconus in rural maharashtra in central India: the central India eye and medical study. Am. J. Ophthalmol.2009;148 (5), 760-765.

10. Michaud, L. V. Predicting estimates of oxygen transmissibility for scleral lenses. Contact Lens and Anterior Eye.2012; 35(6), 266-271.

11. Vessel, M.2020 AAV Media, LLC. Retrieved from www.allaboutvision.com:https://www.allaboutvision.com/conditions/aberrations.html. 